\title{
Article
}

\section{To Sit or Not to Sit in VR: Analyzing Influences and (Dis)Advantages of Posture and Embodied Interaction}

\author{
Daniel Zielasko $^{1, *(\mathbb{D})}$ and Bernhard E. Riecke ${ }^{2, *} \mathbb{C}$ \\ 1 Human-Computer Interaction, Department IV—Computer Science, University of Trier, 54296 Trier, Germany \\ 2 School of Interactive Arts and Technology, Simon Fraser University, Surrey, BC V3T 0A3, Canada \\ * Correspondence: daniel.zielasko@rwth-aachen.de (D.Z.); ber1@sfu.ca (B.E.R.)
}

Citation: Zielasko, D.; Riecke, B.E. To Sit or Not to Sit in VR: Analyzing Influences and (Dis)Advantages of Posture and Embodied Interaction. Computers 2021, 10, 73. https:// doi.org/10.3390/computers10060073

Academic Editor: Paolo Bellavista

Received: 31 March 2021

Accepted: 18 May 2021

Published: 3 June 2021

Publisher's Note: MDPI stays neutral with regard to jurisdictional claims in published maps and institutional affiliations.

Copyright: (c) 2021 by the authors. Licensee MDPI, Basel, Switzerland. This article is an open access article distributed under the terms and conditions of the Creative Commons Attribution (CC BY) license (https:// creativecommons.org/licenses/by/ $4.0 /)$.

\begin{abstract}
Virtual Reality (VR) users typically either sit or stand/walk when using VR; however, the impact of this is little researched, and there is a lack of any broad or systematic analysis of how this difference in physical posture might affect user experience and behavior. To address this gap, we propose such a systematic analysis that was refined through discussions and iterations during a dedicated workshop with VR experts. This analysis was complemented by an online survey to integrate the perspectives of a larger and more diverse group of VR experts, including developers and power users. The result is a validated expert assessment of the impact of posture and degree of embodiment on the most relevant aspects of VR experience and behavior. In particular, we posit potential strong effects of posture on user comfort, safety, self-motion perception, engagement, and accessibility. We further argue that the degree of embodiment can strongly impact cybersickness, locomotion precision, safety, self-motion perception, engagement, technical complexity, and accessibility. We provide a compact visualization of key findings and discuss areas where posture and embodiment do or do not have a known influence, as well as highlight open questions that could guide future research and VR design efforts.
\end{abstract}

Keywords: virtual reality; posture; embodiment; locomotion; systematic analysis; survey

\section{Introduction}

The kind of interfaces and physical setups that have been used for Virtual Reality (VR) and computer gaming applications have in the past been often driven not just by the overall goal and functional and non-functional requirements, but also by the availability, quality, and affordability of technology. For example, even though many computer games simulate avatars as walking, running, or doing more elaborate physical maneuvers (from jumping to various sports), the user is in most cases sitting comfortably and operating hand/finger based controllers (although there are notable exceptions, such as many arcade and exergames). And even if users are standing, their posture and movements are often still quite different than the (typically much more active and whole-body) movements of their avatar. How could such inconsistencies between our physical versus simulated posture and movements affect the naturalism, believability, and overall effectiveness of the simulation and user experience? Are there scenarios where it does not really matter as much if there are inconsistencies? And what are the scenarios where it might be more advantageous and desirable (or not) to be comfortably seated as compared to adopting a more active and engaged posture, such as standing or physically moving around? Given that many of us tend to opt for the more comfortable (typically seated) posture, what are the scenarios where it would be "worth it" and clearly beneficial to get up from our comfy seat to at least stand or even move around? There is surprisingly little research in this area.

Bellgardt et al. [1] briefly discussed related questions for the specific scenario of workplaces and illustrated this with three different use cases (factory planning, automotive design reviews, and immersive graph exploration). As a result, they provided design 
guidelines for the different scenarios (sitting, standing, and walking) regarding the role of immersion, locomotion technique, input devices, session duration, and the inclusion of the environment itself.

Simultaneously, Zielasko et al. [2] provided a detailed review on challenges that occur with and (interaction) techniques that are usable in, a sitting and desk-based working scenario. They concluded inter alia, that locomotion techniques are one of the key differences between a standing and a sitting scenario, while interaction techniques for selection \& manipulation and system control, can benefit from adaptions to the scenario but in most cases work in both.

Di Luca and colleagues [3] recently provided a comprehensive taxonomy and database of different locomotion techniques that include visualizations of different attributes. This taxonomy provides an excellent broad overview and context for the current paper, but it was only based on the evaluations by the authors themselves which had not tried out all the interfaces, and does not (yet) allow for a more detailed analysis or classification of advantage and disadvantages of seated versus standing user posture, and how it might interact with the degree of embodiment of the interfaces, which is the focus of the current paper.

In the current paper, we aim for a more general discussion of the potential pros and cons of seated versus standing posture during VR usage. To this end, we propose a classification of these and conducted a survey amongst VR experts (including researchers, developers, and power users) to gain a deeper understanding and broader perspective that goes beyond the authors' own background and personal experience. This work was inspired by and extends a proposal we made as part of the 1st Workshop on SeatedVR at IEEE VR 2020 [4], where it was actively discussed among the participants. The results were used to adapt and extend the original classification-thanks to all the workshop participants who contributed their insights and ideas!

With this paper, we hope to make contributions towards several interrelated goals:

1. Attempt to provide a broader overview of the characteristics of a wide range of locomotion interfaces and interface types, to provide a more unified representation and classification.

2. Enable VR designers to more easily determine what kind of posture and locomotion interfaces might be most suitable, based on the identified functional and non-functional requirements of the VR experience.

3. Help pinpoint open research/development questions and better understand specific challenges and opportunities, e.g., in most VR use cases, there is arguably a mismatch between the physical setup (e.g., sitting in your office or living room with an HMD on your head and controllers in your hand) and the simulated VR scenario (e.g., sitting in a racing car, or walking around in armor fighting dragons). What aspects of this mismatch are critical or not? How important is it to match one's posture and sit versus stand physically to match the virtual scenario (e.g., of sitting in a racing car versus standing/walking)? Could it be easier to imagine/simulate standing when actually sitting than the other way around?

4. Provide a framework to help tackle open questions in research and development, e.g., which posture to use when and why? Could it be worthwhile to explore hybrid interfaces that combine the advantages of sitting versus standing/walking, and allow for an easier and more seamless transition between the different postures? Or could it make sense to provide flexible interfaces, such as sit-stand stools, like the MovMan [5], or bar stools that make it easier to get up from sitting, as it can provide a much higher sitting posture, which has been observed to be desirable in collaborative design review sessions [6]?

We hope that this work will stimulate further discussion and research, including human participant research that will hopefully be possible again in the near future once the worst part of the current Covid-19 pandemic is over. 


\section{Methods}

\subsection{Iterative \& Qualitative Expert Evaluation and Validation}

We conducted a workshop (1st Workshop on SeatedVR at IEEE VR 2020) to present the initial peer-reviewed evaluation of (dis)advantages of posture in VR [4]. During the workshop, we conducted a designated breakout session where these initial findings were discussed in detail, and feedback and suggestions were elicited in writing and subsequently discussed. Participants suggested several improvements which were used to iteratively refine the description and overview table of the pros and cons of seated versus standing posture until all suggestions were incorporated and a consensus was reached among all participants. The presentation of the results in this manuscript follows these suggestions.

\subsection{Online Survey}

To further guide our analysis and classification, we conducted an online survey where VR experts were asked to rate diverse aspects of different VR locomotion interfaces. Our survey was answered by 61 participants (age $M=37.5, S D=13.2$ ) recruited through various email lists and social media, with at least 2 years of expertise in VR research, development, or usage, and at least 20 experiences in VR. Eight participants specified themselves as female, including transgender women; 47 as male, including transgender men; and 6 preferred not to provide any information. Forty participants specified themselves to have more than 200 experiences in VR, 16 between 51-200, and 5 between 20-50. Participants that specified less than 20 experiences in VR or have used VR for less than two years were excluded from the analysis. The number of years with experience in VR is summarized in Figure 1. Participants were allowed to skip questions, so not all participants answered all questions. The study procedure, including data acquisition and handling, was approved by the ethics board of the University of Trier.

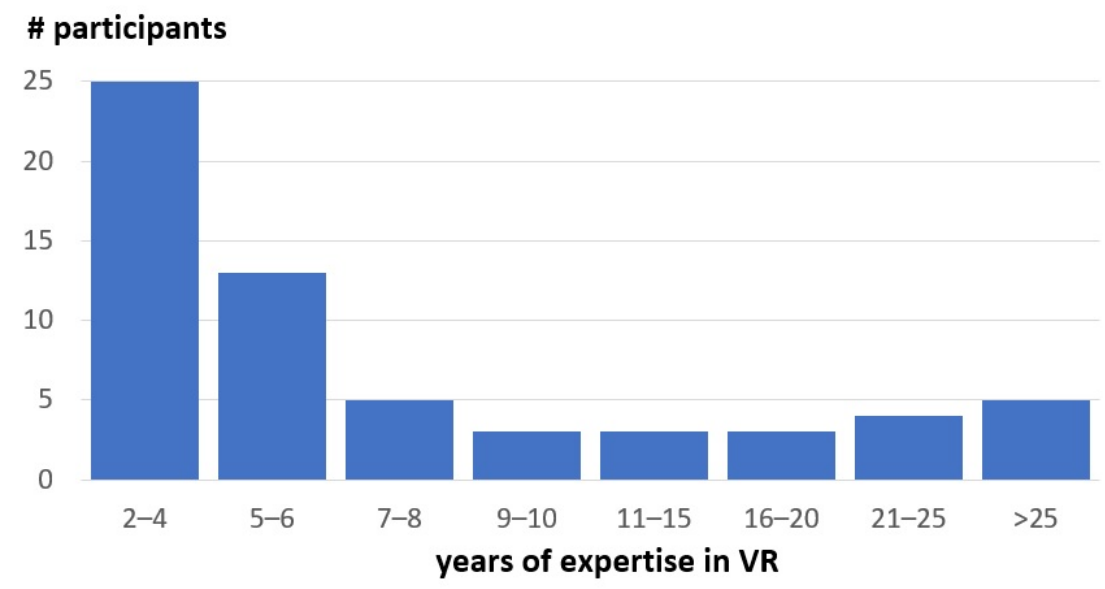

Figure 1. Histogram of survey participant's years of expertise in VR.

\section{Results}

\subsection{Online Survey}

The survey was part of a larger study and did not focus specifically on seated versus standing locomotion, although it included seated versus standing leaning-based locomotion that we will discuss in more detail below. As the focus of the current paper is to discuss the potential effects of posture on user experience and behavior in VR, and how this might be mediated by the degree of embodiment, we present the relevant data from the survey in Figures $2-4$ and discuss it below in more detail. 


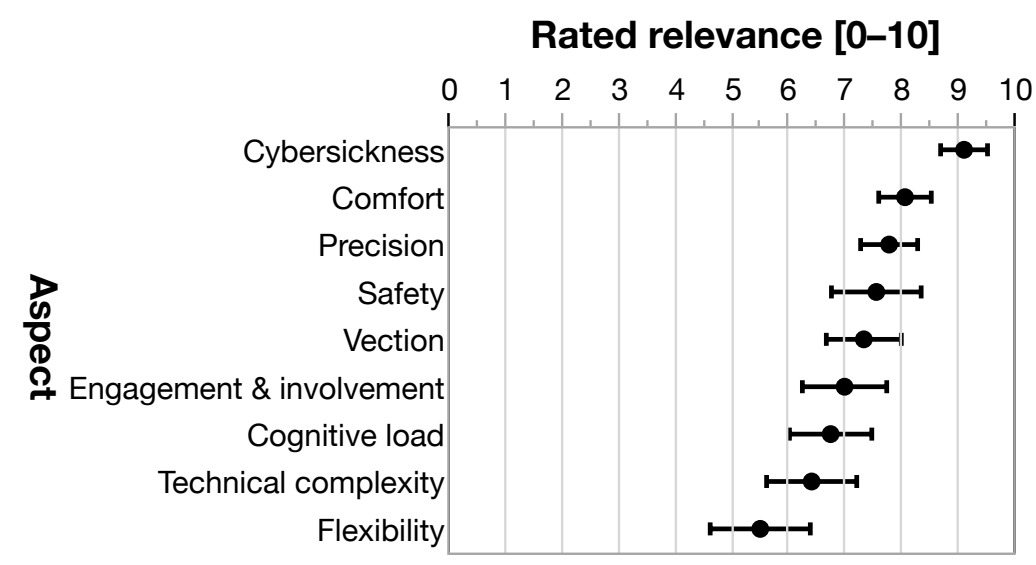

Figure 2. Survey participants' mean ratings of relevance of the different aspects of VR locomotion interfaces, on a scale from $0=$ completely irrelevant to $10=$ crucial/very relevant. Error bars indicate $95 \%$ confidence intervals.

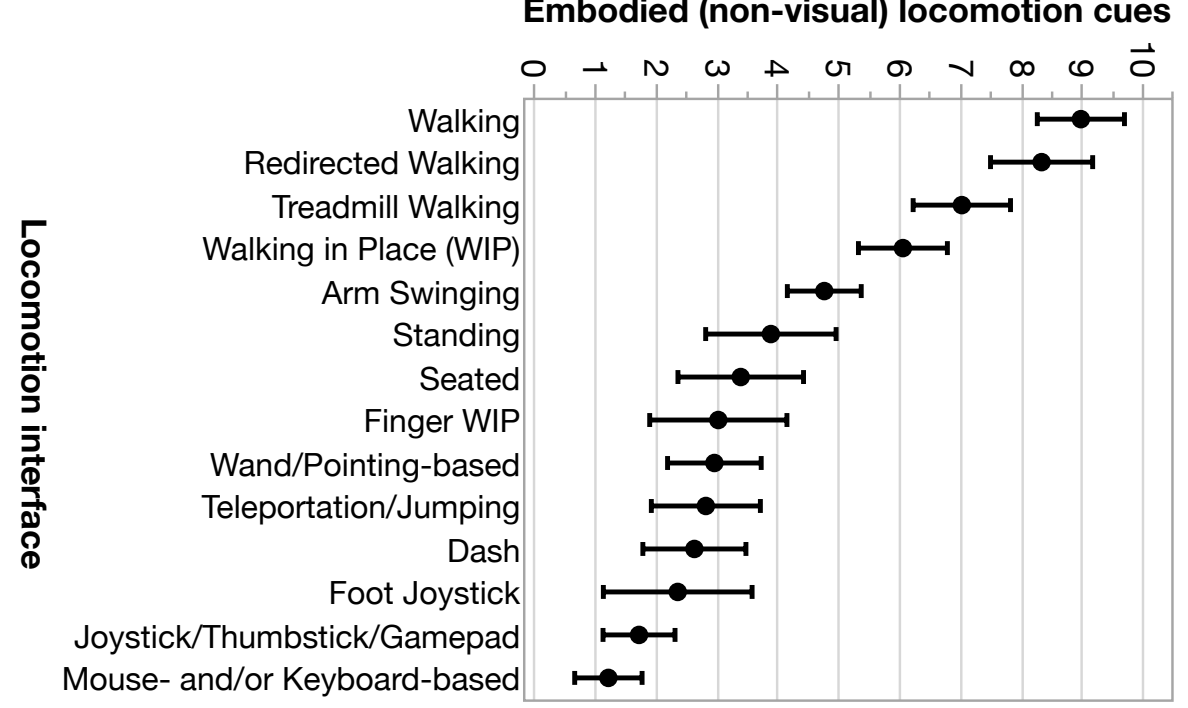

Figure 3. Survey participants' mean ratings of the degree of embodied non-visual locomotion cues for different locomotion interfaces, on a 0-10 ratings scale. Error bars indicate $95 \%$ confidence intervals. While seated and standing leaning-based interfaces were rated separately, for the other locomotion interfaces, this survey did not distinguish between seated and standing/upright interfaces.

\subsubsection{Rated Relevance of the Different Aspects of VR Locomotion Interfaces}

As part of the survey, participants were asked to rate the relevance of different aspects of VR locomotion interfaces on a scale from $0=$ completely irrelevant to $10=\mathrm{crucial} /$ very relevant. The different aspects rated and the results are summarized in Figure 2, sorted by rated relevance. The data clearly shows how the VR experts participating in the survey rated cybersickness to be the most crucial aspect, with a mean of 9.1 on a $0-10$ scale $(S D=1.4)$. In fact, cybersickness was rated as significantly higher than the next highest rated aspect of VR locomotion interfaces, comfort $(p=0.0044)$, which was rated at 8.0 $(S D=1.6)$. Flexibility (which in the survey was defined as "Application Diversity and Flexibility: How flexible/adaptable is the locomotion interface in terms of being suitable for a broad range of different scenarios and applications beyond walking?") was rated as the least relevant aspect of VR locomotion interfaces $(M=5.5, S D=2.7)$, which was significantly below the next lowest rating of technical complexity $(M=6.4, S D=2.4$, $p=0.023$ ). The remaining aspects did not show significant differences compared to their direct neighbors in Figure 2. Note that all aspects were rated as more relevant than the midpoint of 5 on the $0-10$ scale used, indicating that all of them were rated as having 
relevance and should be considered in designing and selecting VR locomotion interfaces, although some were rated as more critical. We will use this rated order of relevance to structure the final classification in Section 3.2.
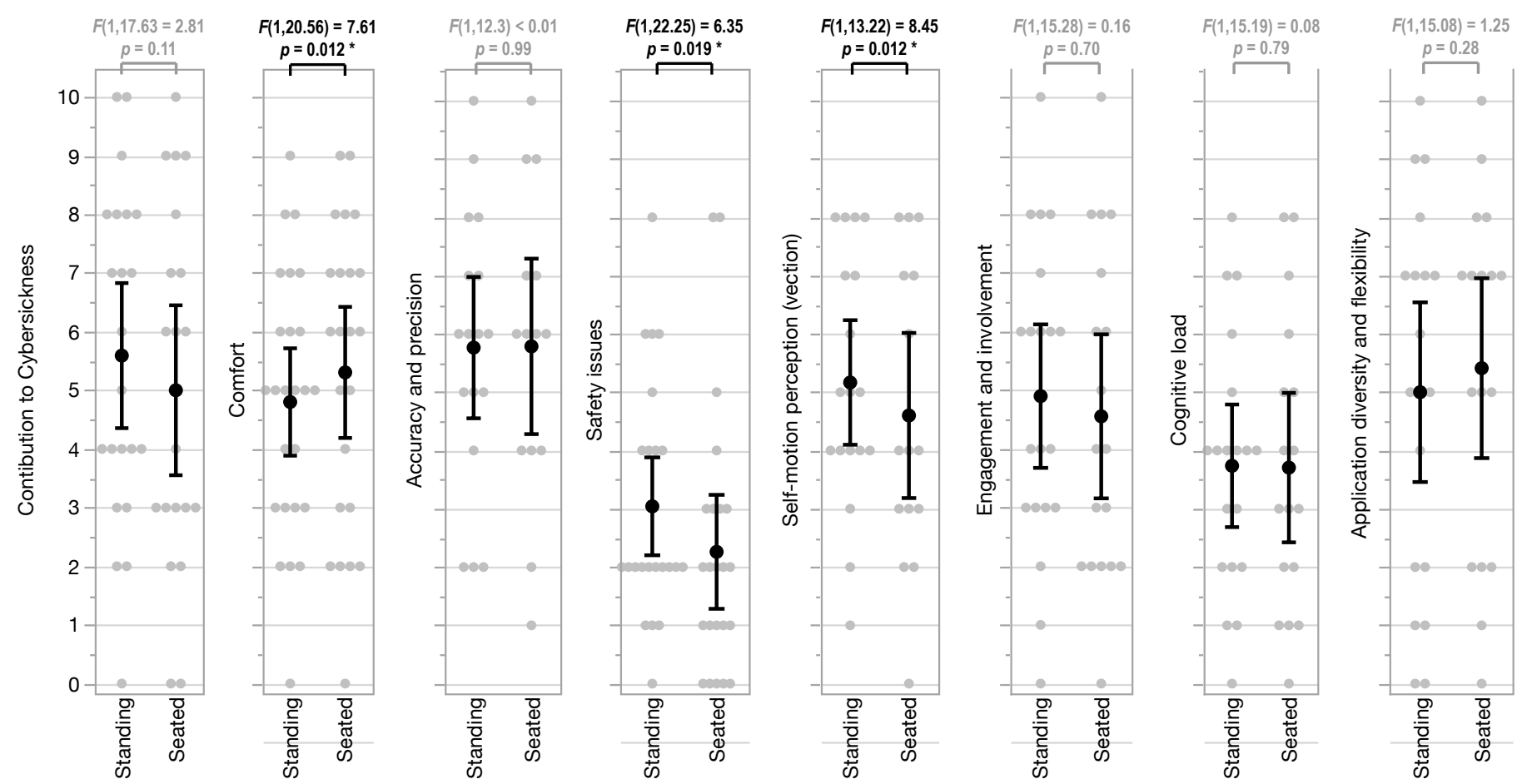

Figure 4. Expert assessment of seated versus standing leaning-based locomotion interfaces with respect to eight user experience and usability aspects, on a scale from 0-10. Error bars depict 95\% confidence intervals, and the gray dots indicate individual participant's ratings to illustrate the often rather wide range and distributions of the ratings. Significant differences are formatted in black text at the top of the respective data plots. Compared to standing, seated leaning-based interfaces were rated to provide significantly higher comfort while reducing safety issues and the perception of self-motion (vection). Note that these ratings were only comparing seated versus standing leaning-based locomotion interfaces, and future research is needed to investigate how these ratings might generalize to other kinds of seated versus standing interface or scenarios.

\subsubsection{Rated Degree of Embodiment of VR Locomotion Interfaces}

Already in the first iteration of the classification [4] that was not based on an online survey, the degree of embodied interaction was identified as an additional factor that is important to include in a systematic assessment of (dis)advantages of posture in VR. Thus, to provide a better and more easily accessible overview of the embodied interaction continuum (see Figure 5) we asked the survey participants to rate a representative group of 14 locomotion interfaces regarding the amount of embodied non-visual locomotion cues provided by the interface. Specifically, they were asked: "How would you rate the amount of involved kinaesthetic cues (awareness of joint and body part movement), proprioceptive cues (awareness of joint and body part positions), or vestibular cues (sense of self-movement and balance, provided by your inner ear) supported by this locomotion interface?" The scale was from $0=$ none to $10=$ very high. The results are given in Figure 3 . 


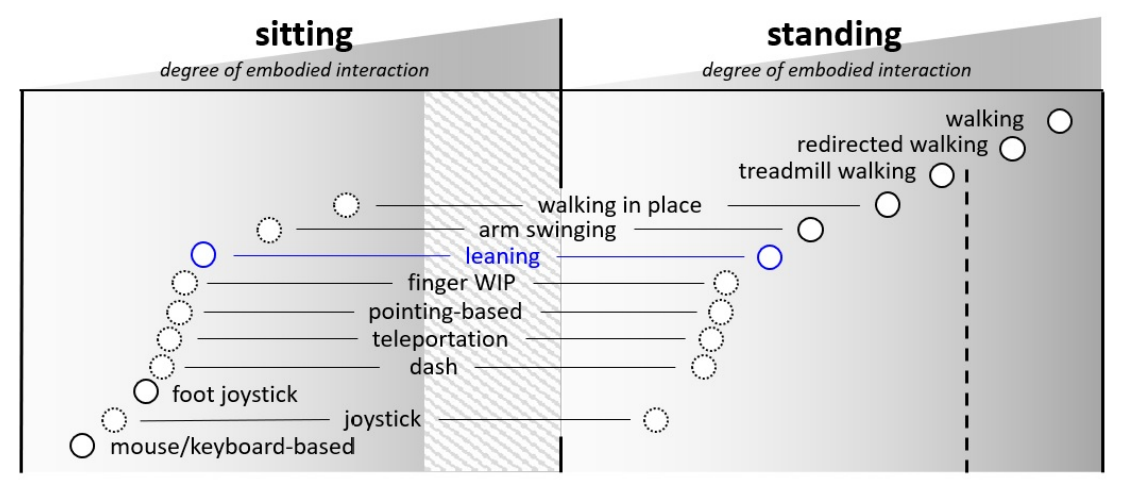

Figure 5. Mean survey ratings of the degree of embodiment (increasing from left to right) provided by different locomotion interfaces, split into seated (left) and standing/upright postures, including walking (right). Ratings for leaning-based interfaces are depicted as blue rings and were the only ones where the survey explicitly distinguished between seated and standing posture. Interfaces were depicted using thick rings if they have a common default posture, such as sitting for foot joystick and mouse/keyboard-based interfaces and standing for arm swinging and walking-based interfaces, including walking in place, as well as treadmill, redirected, and normal walking. This figure aims to illustrate the two main factors of our classification of (dis)advantages: posture (sitting versus standing/upright) and degree of embodiment. This should help, first, to illustrate how the degree of embodied interaction is really a continuum and, second, to support the placement of estimates to known interfaces. We are using locomotion interfaces here for illustration only, taking into account that locomotion is only one part of the possible interaction space (see Section 4.1). The dashed line divides real walking from simulated walking and other less embodied locomotion paradigms. The resulting area of high embodiment for standing interfaces is depicted as a hatched area for sitting interfaces, to illustrate that this high degree of embodied interaction is not reachable when sitting.

\subsubsection{Comparing Standing vs. Seated VR Locomotion Interfaces}

Finally, participants were also asked to rate standing versus seated leaning-based locomotion interfaces with respect to the different aspects described in Section 3.1.1. Results, including the inferential statistics, are depicted in Figure 4. The standing and seated leaning-based interfaces vary only in one factor, namely the posture; thus, the following classification are utilized for a cross-check of the inferred assessments. The experts rated the seated (versus standing) leaning-based locomotion interfaces as significantly more comfortable and having fewer safety issues, but also as less effective in contributing to a compelling sensation of self-motion (vection) (see Figure 4 for the detailed statistics results). There was also a non-significant trend towards seated leaning-based locomotion interfaces contributing less to cybersickness than the standing counterpart. Note, however, that the comparison of seated versus standing in this survey was only for leaning-based locomotion interfaces, and future research is needed to provide a thorough evaluation of how sitting versus standing might influence user experience and behavior for other locomotion interfaces, as well as interactions, which were not covered by this survey.

\subsection{Classification}

In this paper, we distinguish between seated and standing user postures, although the transition can be continuous, e.g., when using flexible seating, such as sit/stand stools. Note that we use the terms seated versus standing here to refer to the general posture of the user in terms of being supported by some kind of seat versus not, i.e., "standing" here explicitly includes walking and other non-seated movements. During our research, we noticed early on that only a few user experience and performance aspects were clearly always positively or negatively affected by the user's body posture alone. Often, the degree of embodiment of the used interaction device/paradigm can play a similarly important role; hence, we decided to add it as a continuous factor to our classification summarized in Figure 5. For instance, whether or not the VR experience comes with 
significant safety concerns differs not only between sitting and standing but also heavily depends on the level of embodiment and user movements, i.e., whether the users are standing stationary, wildly swinging their arms and legs, or even freely walking, running, or performing martial arts moves (see Section 4.4). Different interactions and corresponding degrees of embodiment can range from using merely fingers/hands to control simulated interactions and (self-)motions (e.g., mouse \& keyboard, thumb-sticks/joysticks, trackpads, or gestures), to more embodied interactions (such as leaning-based interfaces, headbased interactions) to diverse walking-based interactions, as detailed in the below sections and figures.

The resulting classification of (dis)advantages with respect to the two factors user posture (sitting, standing) and degree of embodied interaction is summarized in Figure 6. The figure classifies different combinations of posture and degrees of embodiment as advantageous $=+$; neutral $=0$; or disadvantageous $=-$ in comparison to the baseline of a low embodied standing scenario for one dependent variable. We chose a low-embodied, standing scenario as a common reference point and baseline, as it is widely used, well known, and easily accessible to all VR users. Note that the purpose of the simplistic $+/$ - ranking is to provide a simple and easily understood tool to rank and compare combinations among each other and is not intended to describe a linear or absolute metric scale, nor the actual impact size on the corresponding variable, nor is it directly comparable between different variables. There might be variables that are mainly influenced by the users' posture and others only to a much smaller degree. The +/ - ratings serve as a visual shorthand and are discussed in the following section.

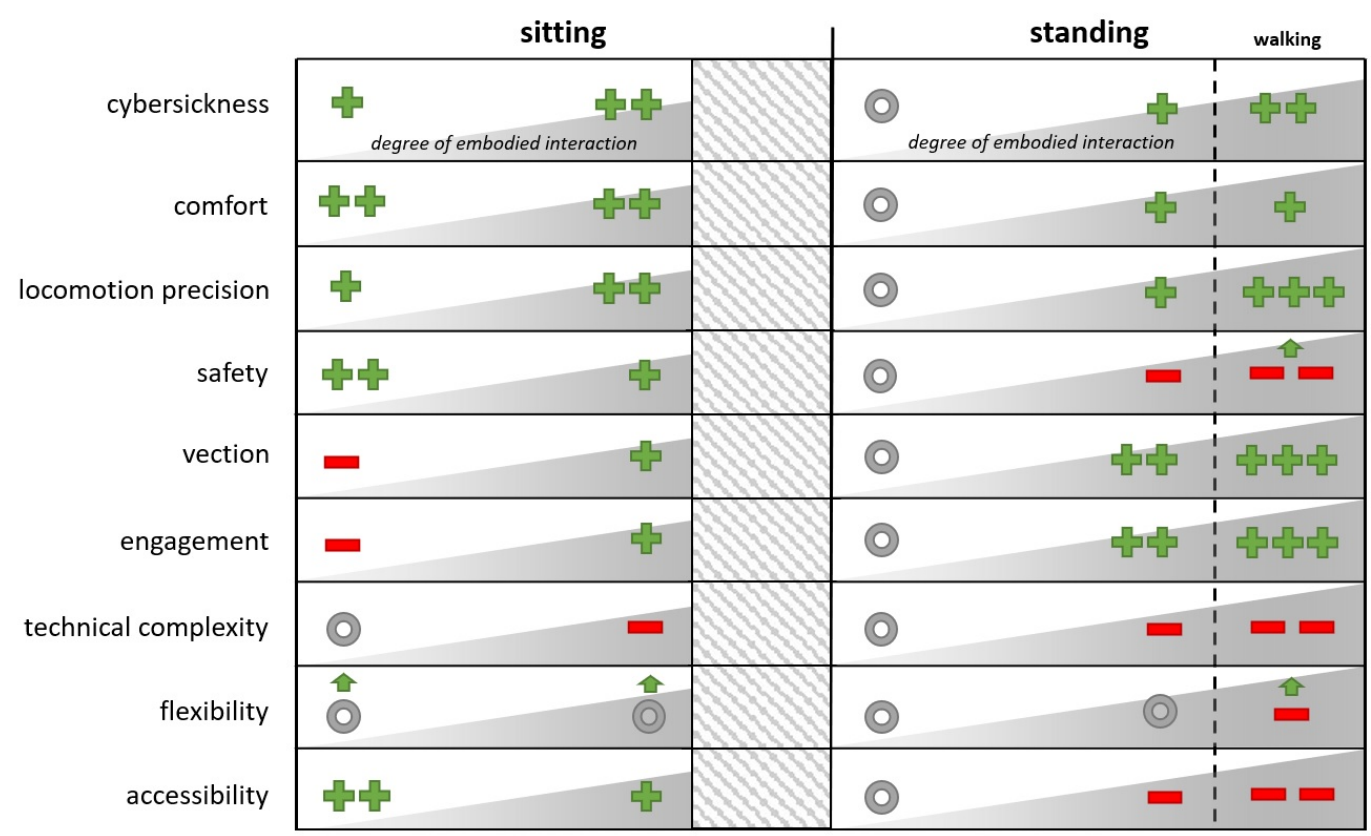

Figure 6. Ranked expert assessment of (dis)advantages of body posture and degree of embodied interaction (see Figure 5) on dependent variables, that are again ranked accounting their relevance (see Section 3.1 and Figure 2), compared to a low-embodied, standing scenario as a baseline. The hatched area depicts a degree of embodied interaction that is not reachable, when sitting, and would have been comparable to walking.

The dependent variables are presented in order of decreasing relevance based on the expert ratings from the survey (see Section 3.1) summarized in Figure 4, starting with the aspect that was rated as most relevant, cybersickness (relevance: $M=9.1, S D=1.4$ ). Additional aspects not covered by the online survey (e.g., accessibility) are listed at the end. As the focus in this paper is on comparing seated versus standing postures for VR, we do not include additional variables that did not seem to have a consistent or systematic impact 
on posture, even if they were rated as overall relevant, such as overall usability (relevance: $M=7.8, S D=2.3)$, spatial orientation $(M=7.6, S D=1.9)$, and learnability of interfaces. Other variables, such as metabolic energy consumption, are not listed, as the impact of posture is obvious, but whether or not a high/low energy consumption is advantageous depends largely on the context, user goal, and application.

Figure 5 provides a visual representation of the degree of embodied interaction afforded by different VR interfaces for seated (left) versus standing/non-seated posture, that serve as the blueprint for the individual categorizations and discussions of (dis)advantages of body posture and embodied interaction for different user experience and usability aspects. To provide a reference point, we added the survey data of locomotion interfaces from Section 3.1 as black or blue circles whenever it was obvious whether the user is considered standing/upright (walking, redirected walking, treadmill walking, walking in place, arm swinging, and standing-leaning based interfaces) or seated (seated leaning-based, foot joystick, and mouse/keyboard). For the remaining interfaces where the survey data is unclear whether it was rated for seated versus standing/upright posture, we illustrated this by dashed circles (finger WIP, pointing-based, teleportation, dash, and joystick). Note that our survey, as well as in Reference [3], only included locomotion interfaces but not other modes of interaction in VR, and there is overall rather limited research evaluating potential benefits and drawbacks of seated versus standing posture and embodiment in VR; hence, in the following, we attempt, such as classification and discussion, using the visual layout of Figure 5 as a blueprint and visual shorthand.

\section{Discussion}

In this section, we will discuss the final classification of (dis)advantages of seated versus standing scenarios, and how they interact with the degree of embodied interaction for the aspects that were rated as most relevant in our online survey, starting with cybersickness in Section 4.1.

\subsection{Cybersickness}

In this work, including the online survey, we separated cybersickness (see Figure 7) from the more general aspect of comfort (see Section 4.2). The connections are complex as cybersickness can certainly contribute to fatigue and discomfort, and not getting sick is a necessary prerequisite to feeling comfortable. Given the potentially drastic negative impact of sickness on the user experience, we treated cybersickness and comfort separately here. Part of the complexity of cybersickness originates in the fact that it is still not fully understood [7], although it is clear that multiple factors contribute and interact, and together contributing to cybersickness (for reviews on cybersickness and underlying factors, see Reference [8-11]). Regarding potential influences of posture, some studies found sitting to be beneficial for reducing cybersickness [12,13] or at least showed a trend in doing so [14]. A comparative study over various embodied and non-embodied steering methods [15], that we are currently repeating in a standing posture but is paused because of Covid-19, is also clearly pointing towards these observations. The experts' assessment in our online survey (see Figure 4) shows a similar trend for reduced cybersickness for seated as compared to standing leaning-based interfaces, but this trend did not reach significance. This tendency for increased cybersickness for standing as compared to sitting VR users might be related to the increased postural instability during standing, which, according to the ecological theory by Riccio and Stoffregen [12], can contribute to motion sickness. However, there are also experiments showing no contribution of posture to cybersickness at all [16], suggesting one or more factors are interacting, or a dependence on the specifics of the simulation and locomotion method. The latter study, for instance, did not involve any actively controlled virtual travel, while the others did, which might at least partially explain the different observations, and warrants future research. It is, however, clear that simply sitting down does not prevent users from getting sick [17], even though it might help with mitigating cybersickness. 


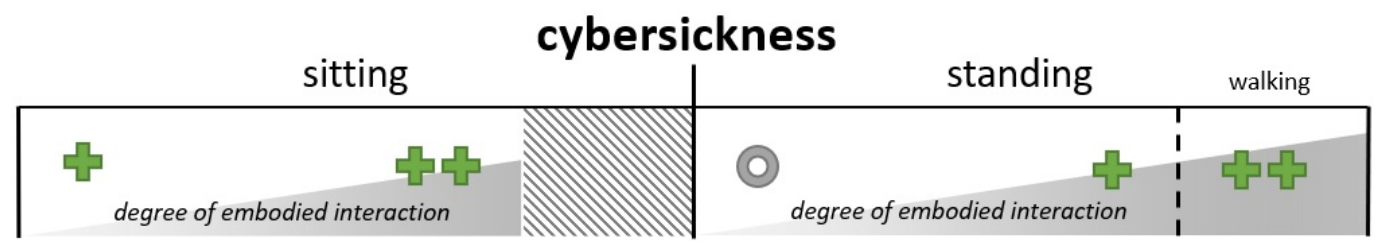

Figure 7. (Dis)Advantages of body posture and degree of embodied interaction (see Figure 5) on cybersickness (relevance: $\mathrm{M}=9.1$ ) compared to a low-embodied, standing scenario as a baseline.

There seems to be no interaction effect between posture and degree of embodied interaction observed yet. In contrast, the degree of embodiment in the locomotion interface seems to independently have a positive impact on the contribution to sickness. This is, compared to using, e.g., hand-based controllers, using more embodied locomotion interfaces, such as leaning-based paradigms that provide at least some vestibular cues during simulated accelerations, can help to reduce cybersickness $[14,18,19]$, potentially due to the reduced inter-sensory cue conflict. This also illustrates that the effect of more embodied interaction is not merely generated by the pure amount of bodily movements and involved body parts, but that the movements necessary to use the interface have to match the simulated self-motions, or otherwise can have the opposite effect [20]. Important to consider is also that many seated VR scenarios do not allow users to rotate freely, for example, when sitting on a couch or a non-turning chair, or environmental factors that prevent rotation (e.g., sitting at an office desk). These situations, thus, often require virtual instead of physical rotation, which can counteract the potential positive effect of the sitting posture, thus increasing cybersickness because of the lack of physical rotation ability. A negative impact of virtual rotation on cybersickness is exemplified in work from Kemeny et al. [21] and Farmani et al. [22].

Note that, in this manuscript, we only discuss the influence of posture and degree of embodied interaction, and there are of course many other factors that can contribute (see, e.g., Reference [3]). For instance, teleportation and pointing-based steering are rarely distinguishable by their degree of embodied interaction, however, also independently of the user's posture the steering method does contribute much higher to cybersickness.

That said, without a complete understanding of cybersickness, it is also difficult to judge to which degree differences in the contribution/mitigation to sickness of posture, are intrinsic, technological, or intrinsic but still solvable by technology in the future, such as with an external vestibular stimulation [23].

\subsection{Comfort}

In this classification, we are considering comfort (see Figure 8) irrespective of cybersickness, which is discussed separately in the previous Section 4.1. The comfort of an interface includes its support for long-term usage, covering, for instance, ergonomics and fatigue. In non-VR environments, sitting has clearly shown to generate less fatigue, discomfort, and leg swelling than standing [24]. Moreover, standing or even better, walking [25] can be beneficial and part of active physiotherapy against illnesses occurring when sitting for long times and in unfavorable postures. This difference between a sitting and standing posture is intrinsic, and VR developers cannot do much about this, other than supporting sitting when comfort is critical. This is also reflected in the experts' assessment of increased comfort for sitting versus standing in VR (see Figure 4). Interestingly, though, fatigue is especially an issue in a standing posture when it is unsupported or for no special reason [26]. Balasubramanian et al. [27] were able to show that fatigue in the leg and lower back muscles during a real-world assembly task was reduced in a dynamic as compared to a more static standard standing posture. For our classification, this means that the comfort of a standing posture might actually increase with a higher degree of body involvement and dynamic embodied movements - of course with the caveat that at some point more physical movement (e.g., exergames) will also lead to increased fatigue, but the user's perception and acceptance might be another, even in these extreme cases. 


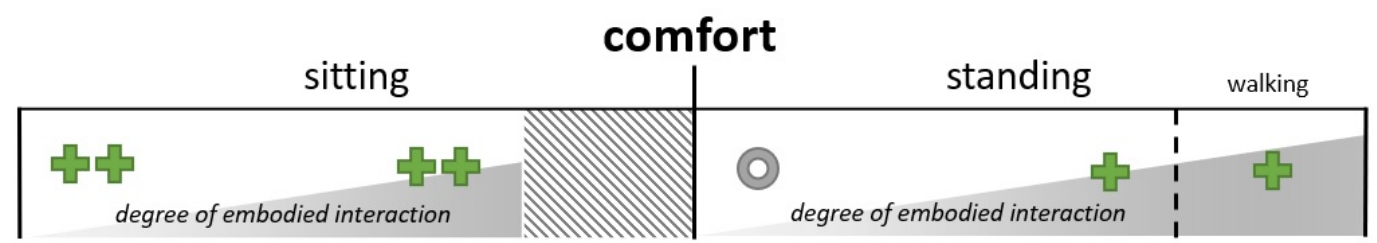

Figure 8. (Dis)Advantages of body posture and degree of embodied interaction on comfort (relevance: $\mathrm{M}=8.0$ ) compared to a low-embodied, standing scenario as a baseline.

\subsection{Locomotion Precision}

VR allows changing laws of physics, starting with something as simple as our scale with respect to the world. Furthermore, the time of movements is elastic, as well as arbitrary transfer functions possible, when using steering techniques (cf. Reference [28]). All this can support or inhibit precision and, thus, the precision of locomotion. For this classification, we accept that this can be similarly applied to all postures. However, given our extensive real-world experience with natural walking, we assume that natural walking in VR with an unaltered one-to-one mapping between physical and virtual movements will achieve the highest possible precision (see Figure 9), while also minimizing cognitive load. As soon a locomotion interface deviates from this one-to-one direct mapping, we argue that a seated (as compared to standing) posture will provide more support and stability, thus allowing for more precise locomotion than its standing counterpart, especially when very fine movements (maneuvering [29]) are needed. However, to the best of our knowledge, none of this has been studied empirically.

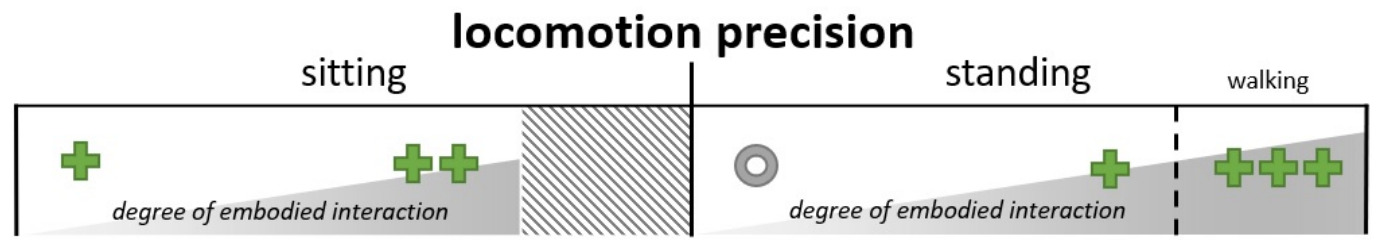

Figure 9. (Dis)Advantages of body posture and degree of embodied interaction on locomotion precision (relevance: $\mathrm{M}=7.8$ ) compared to a low-embodied, standing scenario as a baseline.

While there are some indications for a higher degree of embodiment having a positive influence on locomotion precision $[15,18]$, it is hard to investigate, as the experience with a locomotion interfaces can have huge effects on performance, and especially for low embodied interfaces, like joysticks and gamepads, in which many participants have thousands of hours of experiences of using them, which makes fair comparisons to novel (e.g., more embodied) locomotion interfaces challenging, and potential effects of embodiment hard to isolate in empirical studies. For locomotion precision, the experts' assessment did not indicate a significant effect of posture at least for leaning-based locomotion interfaces (see Figure 4), though, and future carefully designed research is needed to better understand the potential effects of posture and embodiment on locomotion precision.

\subsection{Safety}

Sitting is undoubtedly safer than standing while using VR, as it reduces imbalance and prevents falling over (see Figure 10). Additionally, both sitting and standing VR usage can become less safe with an increasing degree of embodied interaction, although this effect can be more pronounced when standing as discussed below. Additional safety concerns arise when the user can physically move around and potentially bump into obstacles (especially if they are occluded by the HMD and not virtually rendered). There are also potential interaction effects between posture and degree of embodiment on safety, which, for example, shows in locomotion methods that utilize upper body inclination/leaning. Leaning the upper body when standing can create more imbalance and massively increases the risk of falling over, which is less of a concern in a seated scenario. Recent research 
also found anomalies in postural stability right after an HMD-based VR experience and recommended to sit down for two minutes when tending to fall [30]. Thus, it could be the posture, the degree of embodied interaction, or both that also led the VR experts' in our survey to judge a seated leaning interface as safer than an upright one (see Figure 4).

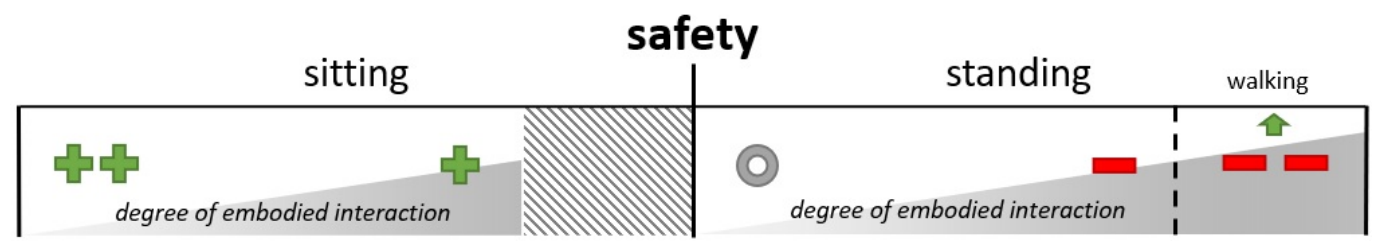

Figure 10. (Dis)Advantages of body posture and degree of embodied interaction on safety (relevance: $\mathrm{M}=7.5$ ) compared to a low-embodied, standing scenario as a baseline. An arrow indicates a potential trend.

The effect of posture and physical movements on user safety in VR is intrinsic, although these safety concerns will likely be reduced by future technology developments; recent advances in chaperones and see-through options are just some examples of such developments that are crucial for the commercial success of and wider applicability of VR. On the other hand, higher perceived safety will likely also reduce users' fear to move, which could result in more accidents if the perceived safety is not backed up by actually increased safety. At the least, improved safety systems should be designed help to prevent more serious injuries and limit remaining risks to a manageable and acceptable level. So far, research has largely ignored the topic of safety issues in uncontrolled and dynamically changing locomotion spaces (sometimes referred to as the cat problem, illustrating the worst case of dynamically moving obstacles), even though such "cat problems" will get more and more common with the rise of consumer VR and untethered inside-out tracking HMDs. Nevertheless, it seems to be both possible and necessary to create appropriate detection and warning systems in the future. More challenging, in our opinion, is making people aware of the limitation of their available movement spaces fast enough (or permanently) [31], such that they trust the system and really make use of the available space, on the one hand, while simultaneously not inducing breaks in presence or interfering with their actual experience and tasks, on the other hand. One promising solution for this critical challenge is virtual substitutions of physical obstacles [32,33], or creating a virtual world that is completely generated based on the physical world [34], with both approaches being not easily applicable in general or in all scenarios.

\subsection{Vection (Perceived Self-Motion)}

When we move through our physical environment, multiple sensory cues contribute to an embodied sensation of self-motion. In VR, when we are not physically moving, under certain conditions, we can still experience a compelling embodied illusion of selfmotion known as vection, especially if provided with wide-field-of-view optic flow, auditory flow, or biomechanical/proprioceptive cues suggesting self-motion (for reviews, see Reference [35-38]). However, vection typically has an onset latency of several seconds, and only gradually increases over time. Providing at least some minimal physical motion cues (e.g., using motion cueing in vehicle simulation) and, in particular, vestibular cues have been shown to significantly enhance self-motion perception [39-42]. Although we are not aware of any published studies explicitly comparing vection in standing versus seated VR users, research has shown that leaning-based interfaces can enhance vection [19,43-45]. Hence, we hypothesized that leaning-based interfaces that provide a larger leaning range could provide earlier and more compelling vection; thus, standing and other postures that provide more movement possibilities and embodied self-motion cues might also help to enhance vection, as illustrated in Figure 11. When comparing seated versus standing leaning-based locomotion in a currently ongoing study, we indeed observed increased vection for standing users. Moreover, providing a cognitive-perceptual framework of 
"movability" where users believe and/or sense that actual self-motion is possible can also enhance vection [38,46-48]. We posit that standing and other non-seated postures will typically provide a more active pose and suggest more movability, which, in turn, might enhance vection. These hypotheses are corroborated by the experts rating standing leaning-based interfaces as more likely to enhance vection than seated ones; see Figure 10.

\section{vection}

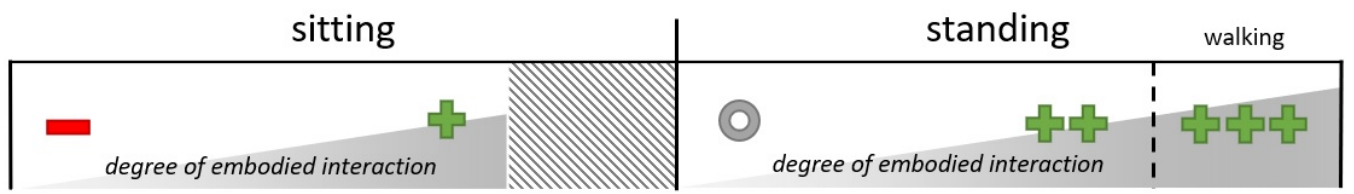

Figure 11. (Dis)Advantages of body posture and degree of embodied interaction (see Figure 5) on users' perceived self-motion (vection) (relevance: $\mathrm{M}=7.3$ ) compared to a low-embodied, standing scenario as a baseline.

\subsection{Engagement \& Enjoyment}

We classified engagement and enjoyment as much more driven by the degree of body involvement than by the posture (see Figure 12). In fact, more embodied interfaces have been shown to generate more enjoyment, engagement, and realism $[49,50]$. Nevertheless, we also propose that there might be an effect of posture, where sitting could feel more passive than standing, and users might more likely take on or perceive to be in the role of an observer-especially if there is little embodied interaction. It is hard to say if this effect is completely intrinsic, as there seem to be solutions to increase the engagement of seated users [51], and it is not clear if this effect holds when the physically seated users actually think that they are standing or walking [52]. However, there will be always limits, and we dare to claim that there is no way seated users will ever perceive themselves running over a virtual tennis court the same way they would if they would physically move around in a large-enough free-space VR walking area, without direct manipulation of the brain.

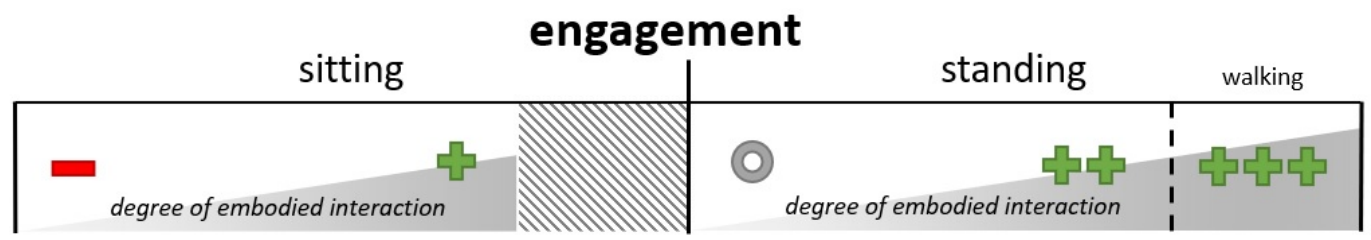

Figure 12. (Dis)Advantages of body posture and degree of embodied interaction on engagement \& enjoyment (relevance: $\mathrm{M}=7.0$ ) compared to a low-embodied, standing scenario as a baseline.

Interestingly, the experts asked in the survey did not see a clear impact of posture on engagement and enjoyment when limited to comparing leaning interfaces (see Figure 4). We would rate the degree of embodiment and engagement with a leaning interface generally as relatively high; therefore, a ceiling effect could have contributed to this lack of a significant effect (even though there was a trend in the predicted direction). Interestingly, however, experts in the survey rated both interfaces to be only in the medium range of engaging and involving in Figure 4. Additionally, both leaning interfaces' degree of embodied interaction was in the survey not rated as high as we would have expected, e.g., less than arm swinging and only barely higher than finger walking-in-place (see Figure 5). These relatively low ratings of leaning-based interfaces might be related to most survey respondents only having limited experience with those interfaces, and it would be interesting to compare ratings after actual usage of different interfaces. We will have a broader discussion on this in Section 5. 


\subsection{Cognitive Load}

There seems to be no clear picture of the direct impact of posture on cognitive performance [53]. One indirect effect that we would expect is related to safety, though (see Section 4.4). Namely, we posit that a seated person might need to worry less about their physical safety or bumping into things and, thus, can forget more about their physical surroundings and be less distracted from the task/experience at hand, as well as experience fewer breaks in presence, which, in turn, might help to reduce cognitive load and divided attention. For the degree of embodiment, expectations are also mixed. On the one hand, more embodiment could lead to more worries about one's safety and the physical surroundings. On the other hand, depending on the kind of embodiment, more embodied interactions potentially more closely mimic real-world interactions and, thus, might be more familiar and automatized (e.g., for physical walking), which can help to reduce cognitive load. Note, however, that our examples of seated and standing leaning-based locomotion interfaces are not really closer to real-world interaction nor very familiar, even though they do generate less vestibular conflicts than traditional hand-controller continuous locomotion methods. Still, Zielakso et al. [15] found fewer environmental collisions with leaning interfaces compared to controller interfaces in a dual-task design, which might relate to cognitive load but also could be explained with more body awareness. Moreover, participants in Nguyen-Vo et al. [14] reported reduced mental workload when performing a navigational search task (where they had to search for eight objects hidden amongst 16 randomly positioned boxes) with a standing leaning-based interface (NaviBoard) or Walking, compared to using a hand-held controller, whereas a seated leaning-based interface (NaviChair) showed only a non-significant trend to reduced workload. This suggests that increased embodiment, at least when integrated well, might indeed help to reduce cognitive load, whereas the effect of posture might be more subtle or only indirect. This is confirmed by the expert ratings in our survey, which, at least when comparing leaning interfaces, did not see any clear impact of posture on cognitive load (see Figure 4).

\subsection{Technical Complexity}

There are different aspects of technical complexity (see Figure 13), which each may have a different impact on different users or developers. Different aspects include the complexity of the initial installation, e.g., of an optical room-mounted tracking system, the requirements of a large-enough and safe walking space free of obstacles, the pure amount and costs of gear needed, calibration steps and gear mounting that is necessary every single time a system is used, and, finally, the complexity of methods and algorithms necessary to implement for a given setting. It should be clear that especially the need in hardware and also computational methods often increase with the degree of embodied interaction, especially when this raises additional safety considerations, but the good news is that most of this is not much affected by the posture of the user, although there can be exceptions. For example, full-body motion capture/tracking can, on the one hand, be more challenging in a sitting scenario when the seat and different body parts occlude each other. On the other hand, in a seated scenario, the tracking volume is more clearly defined and smaller than in a standing or walking scenario. Thus, technical complexity and potential effects of embodiment and especially posture are highly context- and application-specific and need to be evaluated on a case-by-case basis.

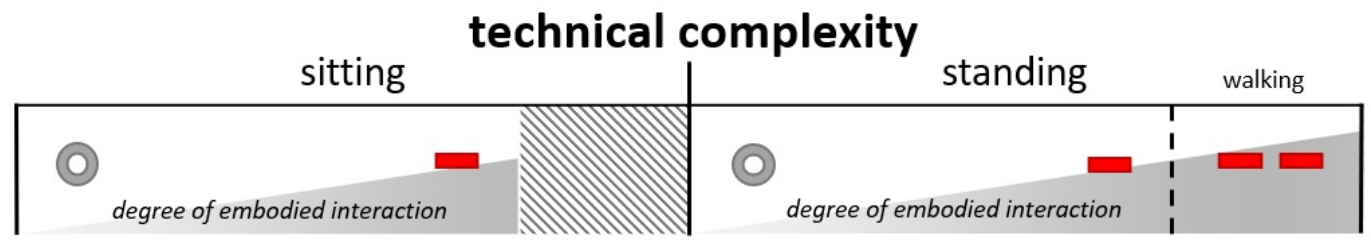

Figure 13. (Dis)Advantages of body posture and degree of embodied interaction on technical complexity (relevance: $M=6.4$ ) compared to a low-embodied, standing scenario as a baseline. 


\subsection{Application Diversity and Flexibility}

In our online survey, we defined "Application Diversity and Flexibility" as follows: "How flexible/adaptable is the locomotion interface in terms of being suitable for a broad range of different scenarios and applications beyond walking? For instance, flying in a plane or spaceship or as a bird or superman/woman, crawling, jumping, swimming, controlling different vehicles (in/underwater, on ground, in air/space), moving the camera for minimally invasive surgery or telepresence, controlling a horse/dragon you're riding, etc." Survey participants rated seated and standing leaning-based interfaces fairly similarly (see Figure 4), with only a small and insignificant trend for increased application diversity and flexibility for the seated condition.

In general, we would expect that a standing posture should better support standing activities in VR, and conversely, a sitting posture should better support simulated seated activities in VR (see Figure 14), such as driving vehicles, etc. Nevertheless, many VR experiences do involve postural mismatches, and it is a very common scenario trying to convince a seated user that they are walking or otherwise moving around [52]. There is surprisingly little research investigating the potential effects of such a postural mismatch, and to what degree users accept or even believe that they are standing or even walking when they are merely seated-or the other way around. On the one hand, there are is a long tradition of seated gaming rarely questioning the miss-match, maybe because sitting is just so much more comfortable and feasible especially in a home setting and for longer exposures. On the other hand, it seems to be pretty clear that we cannot convincingly simulate a $100 \mathrm{~m}$ run, rock climbing, or playing soccer for a seated user.

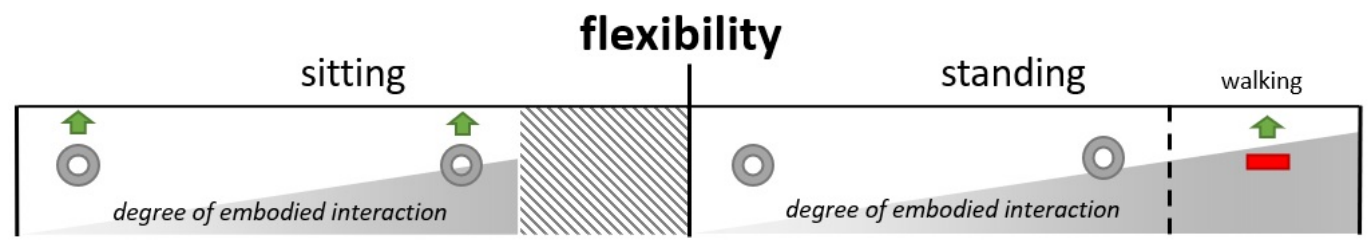

Figure 14. (Dis)Advantages of body posture and degree of embodied interaction on postural independence and flexibility (relevance: $M=5.5$ ) compared to a low-embodied, standing scenario as a baseline. An arrow indicates a potential trend.

At this point, we are only hypothesizing that it is likely easier to make sitting people think/feel/believe or at least accept they are walking in VR than the other way around. The reason for this is that, when it comes to stimulation, it should be easier to add sensations (including the sensation of self-motion), such as generated by muscle activity, optical flow, or airflow, than to remove them. Other factors seem to be already independent of the posture; for instance, Coomer et al. found no difference in spatial awareness [54]. In this classification, we rated walking as having slightly reduced application diversity and flexibility than standing or sitting, as it often requites additional technical setup (e.g., tracking, wireless/wired connection to HMD) and requires a large-enough free-space walking area. However, with the increased availability, affordability, and quality of wireless connections and especially inside-out and hybrid tracking systems and real-time obstacle detection, we expect at least the technical challenges to be largely reduced in the near future. However, knowing that the user is restrained to a limited area might still have advantages compared to a walking user who could still ignore all warning and safety measures and explore areas not intended to be walked in. Specifically, postural flexibility and addressing the user's desire to take a seat or stand up on their own accord could be implemented more easily for standing than walking users, e.g., by having a physical chair nearby but only displaying it virtually when the user intends to sit down.

\subsection{Accessibility}

Today, accessibility gains more and more importance, as society does not only get older on average but also cares more about individuals, including people that are restricted 
in their movements or abilities. Thus, we include here a discussion of accessibility (see Figure 15) even though it was not part of the online survey. There might be a few exceptions, but in general, seated interfaces are more accessible than their standing counterparts. A lot of seated interfaces, for instance, are accessible without any changes, to people with an impairment of the lower limbs or problems to stabilize themselves in a standing posture (which also related to safety, see Section 4.4). Others interfaces are by design constructed for special cases and needs, like the use with one's own wheelchair [55]. However, there are also approaches to support people with balance impairments utilizing specific manipulations of the virtual world and its reactions, such as a static rest frame [56]. Unfortunately, accessibility typically decreases with the degree of embodied interaction, again almost independently from the posture. Hence, it is challenging to design a universally accessible yet highly embodied interface: Either, there is no embodied interaction at all, or a lot of people will be excluded no matter which body part is used for even minimal interaction. Thus, designing a universal interface that is both highly embodied interface and highly accessible seems difficult if not impossible. Instead, designing for accessibility clearly benefits from creating more flexible, modular, and configurable that can be customized and have a low-embodied-interaction and safely seated option as a safe fallback.

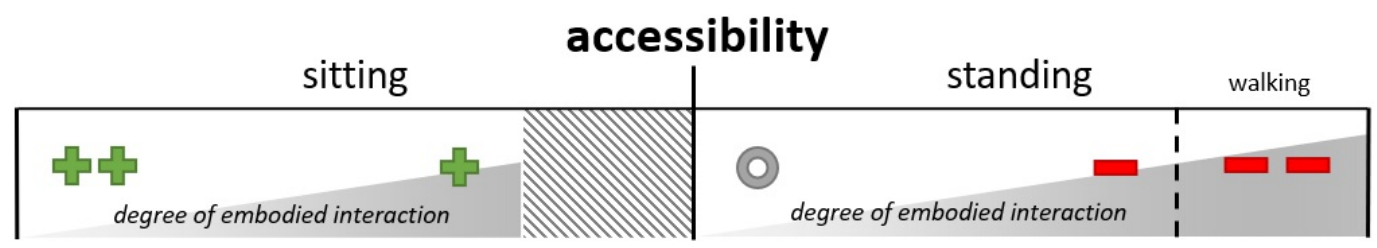

Figure 15. (Dis)Advantages of body posture and degree of embodied interaction on accessibility compared to a low-embodied, standing scenario as a baseline.

\section{General Discussion}

Sitting or standing while being in VR is obviously different, and performing a formal classification on (dis)advantages of those might at first glance seem to be trivial. But apart from some obvious effects of sitting, such as increased comfort and safety, the structural classification in this paper often pointed to more open research questions than clear answers, including some areas where our own analysis did not align with the experts assessment from the online survey. Together, this analysis highlights several interesting research avenues.

As part of our evaluation of the impact on posture on VR user experience and behavior, we discussed whether these differences are purely intrinsically motivated or there is room for technological improvement. Areas where we discussed the potential for such technical improvements that will improve our ratings include safety issues of highly embodied interfaces and postural flexibility.

During our formalization of potential influences of posture, we observed early on a strong dependence on embodiment (cf. Reference [4]). Based on the current analysis, compared to posture, embodiment indeed seems to often have a stronger influence on a lot of the dependant variables (e.g., safety, engagement, technical complexity, and accessibility), which is an important realization and might help to guide future interface development. However, we also discussed that the two factors posture and degree of embodiment are often not independent, but can potentially interact and moderate each other's influence. For example, when standing in VR, more embodied interactions can provide additional safety concerns that are less present when being seated. This interaction is also one of several reasons that we see for the experts' assessments on the example leaning interfaces partially varying from our more general assessment, as aspects of embodiment were not separately assessed in the survey when comparing seated versus standing leaning-based interfaces, and might be specific to leaning-based interfaces.

Furthermore, in the survey, the experts' assessment was often based on extrapolation or pure expectation rather than their own actual experiences with a given interface. 
For example, in the online survey experts assessed their personal experience with leaningbased locomotion interfaces as overall quite low, with a mean of $\mathrm{M}=1.6(\mathrm{SD}=2.7)$ for seated and $\mathrm{M}=1.6(\mathrm{SD}=2.5)$ for standing leaning-based interfaces, on a scale from $0=$ not at all to $10=$ very often. Moreover, and more generally, one's evaluation of a given locomotion interface can heavily depend on the specific implementation-in particular, later and improved implementations might yield more favorable assessments, as we have observed ourselves for the example of leaning-based interfaces. Finally, for a lot of variables, there is limited or no experimental data available; thus, expert evaluations in the survey were necessarily often based on their own experience with these interfaces-or their expectation or intuition when they had no or little experience with a given interface. Indeed, survey participants often differ substantially in their assessments and often spanned the whole response range from 0-10, as indicated by the gray dots in Figure 4.

In the current paper, we used expert evaluations to be able to assess a much broader range of factors and interfaces than would be feasible to experimentally investigate (especially during a pandemic). However, one limitation of this reliance on expert evaluations is that not all of our survey respondents had experienced each of the interfaces. To investigate how large this potential impact of prior experience with a given locomotion interface might be, we compared survey participants' ratings of the amount of embodied locomotion cues provided by the different interfaces depending on whether they had no experience at all with a given interface or at least some experience. Results are summarized in Figure 16, presented similarly to the original embodiment ratings for all participants in Figure 3 but split by experience with the interface. Overall, Figure 16 shows remarkably similar embodiment ratings, irrespective of their experience with an interface.

As illustrated by the gray individual participant dots in Figure 16, however, there were some (more common) interfaces where we had only a few participants who had not experienced them at all, including walking with an HMD in VR $(\mathrm{N}=3)$, arm swinging $(\mathrm{N}=2)$, Wand/pointing-based steering $(\mathrm{N}=3)$, Dash $(\mathrm{N}=3)$, or mouse/keyboard-based locomotion interfaces $(\mathrm{N}=3)$. All participants reported having experience with teleportation, as well as Joystick/Thumbstick/Gamepad in VR, corroborating their widespread usage. For the remaining seven locomotion interfaces, we had at least 5 participants in both the no-experience and at-least-some-experience group, and we ran pairwise comparisons for each of those interfaces to investigate potential effects of prior experience with an interface. Results showed no significant differences between having no or at least some experience for any of these interfaces, all $p^{\prime} s>0.18$. Together with the overall similar response patterns whether or not participants had experienced an interface (cf. Figure 16), this suggests that VR experts with sufficient overall experience with VR (for this study, this was defined as having at least 2 years of expertise in VR research, development, or usage, and at least 20 experiences in VR) seem as a group able to even judge interfaces that they have never tried themselves similarly to those who have tried them at least once. This suggests that expert ratings can be useful and reliable/valid, even when they have no prior direct experience of an interface; however, from the current data, it is not clear on what basis they made there assessments, e.g., whether they imagined using the interfaces displayed in the interface demonstration videos, or whether they based their assessment on their understanding of the literature or others' reports on using such interfaces. Furthermore, future research is needed to further investigate how prior experience affects user ratings also for more diverse aspects/measures and larger and more diverse participant groups, and the decision process underlying assessments of interfaces without being able to actually try them out. 


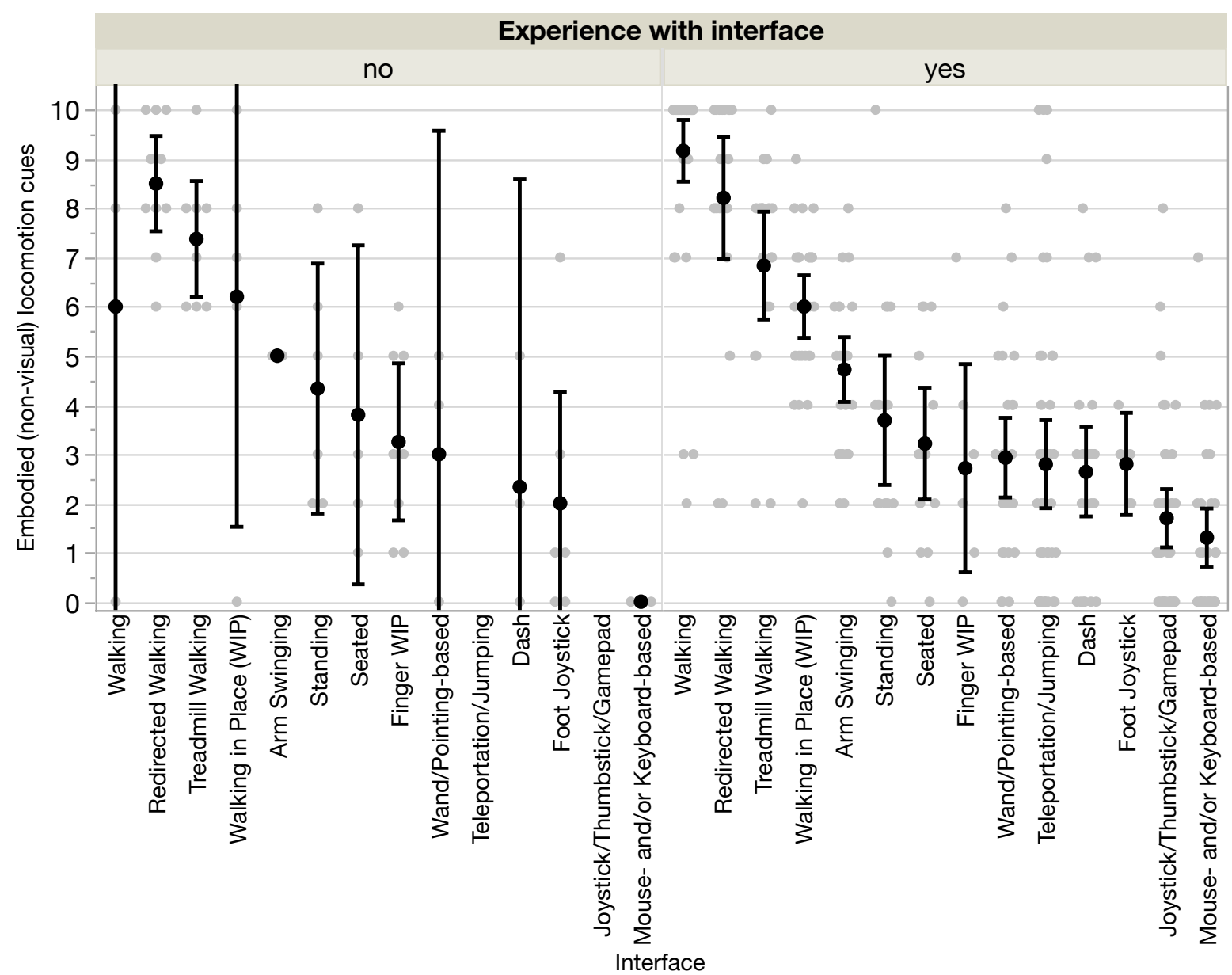

Figure 16. Survey participants' mean ratings of the degree of embodied non-visual locomotion cues for different locomotion interfaces, on a 0-10 ratings scale, split by having no experience at all with the interface (left) or at least some experience with the interfaces (right). Error bars indicate 95\% confidence intervals. Gray dots indicate individual participant ratings.

\section{Conclusions}

In this work, we refined and proposed a classification that shows the impact of user posture and degree of embodiment on relevant aspects of a VR user experience and behavior. The classification aims to serve multiple use cases. First, we hope this paper helps to highlight and explain the areas where posture and the degree of embodiment have a clear effect, to help VR designers and applications focus on key areas. Second, through the comparison and discussion of our own evaluations with the expert evaluations and the literature, we hope to pinpoint some open research questions and potentially promising avenues for future research and development that, in turn, can help to provide a deeper understanding of underlying factors and contributions, which can eventually help further improve VR system design. In the end, no matter what the research findings indicate, people will often have a personal and situation-specific preference. In many situations, however, they will tend to want to sit when experiencing virtual reality [57]. Hence, they will often virtually walk or otherwise virtually move while being physically seated. Therefore, it is key to pinpoint and better understand the implications and challenges of physically sitting in VR in comparison to standing and walking [26], so we can successfully tackle them in future research and development. And as our analysis showed, user engagement seems to be a key factor in this case.

Author Contributions: The authors D.Z. and B.E.R. contributed equally to, data curation, formal analysis, investigation, methodology, visualization, writing — original draft, and writing-review \& editing. All authors have read and agreed to the published version of the manuscript. 
Funding: This research received no external funding.

Institutional Review Board Statement: The study was conducted according to the guidelines of the Declaration of Helsinki, and approved by the Institutional Ethics Committee of the University of Trier (No. 17/2020, date of approval 08/31/2020).

Informed Consent Statement: Informed consent was obtained from all participants involved in the study.

Data Availability Statement: The data presented in this study are available on request from the corresponding author.

Conflicts of Interest: The authors declare no conflict of interest.

\section{References}

1. Bellgardt, M.; Pick, S.; Zielasko, D.; Vierjahn, T.; Weyers, B.; Kuhlen, T.W. Utilizing Immersive Virtual Reality in Everydaywork. In Proceedings of the IEEE VR Workshop on Everyday Virtual Reality, Los Angeles, CA, USA, 19 March 2017 ; pp. 1-4. [CrossRef]

2. Zielasko, D.; Weyers, B.; Bellgardt, M.; Pick, S.; Meißner, A.; Vierjahn, T.; Kuhlen, T.W. Remain Seated: Towards Fully-Immersive Desktop VR. In Proceedings of the IEEE VR Workshop on Everyday Virtual Reality, Los Angeles, CA, USA, 19 March 2017; pp. 1-6. [CrossRef]

3. Luca, M.D.; Seifi, H.; Egan, S.; Gonzalez Franco, M. Locomotion Vault: The Extra Mile in Analyzing VR Locomotion Techniques. In Proceedings of the ACM 2021 CHI Conference on Human Factors in Computing Systems, Yokohama, Japan, 8-13 March 2021.

4. Zielasko, D.; Riecke, B.E. Sitting vs. Standing in VR: Towards a Systematic Classification of Challenges and (Dis)Advantages. In Proceedings of the IEEE VR Abstracts and Workshops, Atlanta, GA, USA, 22-26 March 2020; pp. 297-298. [CrossRef]

5. Kitson, A.; Hashemian, A.M.; Stepanova, E.R.; Kruijff, E.; Riecke, B.E. Comparing Leaning-based Motion Cueing Interfaces for Virtual Reality Locomotion. In Proceedings of the IEEE Symposium on 3d User Interfaces (3DUI), Los Angeles, CA, USA, 18-19 March 2017; pp. 73-82.

6. Freiberg, J. Experience Before Construction: Immersive Virtual Reality Design Tools for Architectural Practice. Master's Thesis, Simon Fraser University, Surrey, BC, Canada, 2015.

7. Chang, E.; Kim, H.T.; Yoo, B. Virtual Reality Sickness: A Review of Causes and Measurements. Int. J. Hum. Comput. Interact. 2020, 36, 1658-1682. [CrossRef]

8. Kemeny, A.; Chardonnet, J.R.; Colombet, F. Getting Rid of Cybersickness: In Virtual Reality, Augmented Reality, and Simulators; Springer International Publishing: Cham, Switzerland, 2020; [CrossRef]

9. Rebenitsch, L.; Owen, C. Review on cybersickness in applications and visual displays. Virtual Real. 2016, 20, 101-125. [CrossRef]

10. Weech, S.; Kenny, S.; Barnett-Cowan, M. Presence and Cybersickness in Virtual Reality Are Negatively Related: A Review. Front. Psychol. 2019, 10. [CrossRef] [PubMed]

11. Keshavarz, B.; Riecke, B.E.; Hettinger, L.J.; Campos, J.L. Vection and visually induced motion sickness: How are they related? Front. Psychol. 2015, 6. [CrossRef] [PubMed]

12. Riccio, G.E.; Stoffregen, T.A. An Ecological Theory of Motion Sickness and Postural Instability. Ecol. Psychol. 1991, 3, 195-240. [CrossRef]

13. Merhi, O.; Faugloire, E.; Flanagan, M.; Stoffregen, T.A. Motion Sickness, Console Video Games, and Head-Mounted Displays. Hum. Factors 2007, 49, 920-934. [CrossRef] [PubMed]

14. Nguyen-Vo, T.; Riecke, B.E.; Stuerzlinger, W.; Pham, D.M.; Kruijff, E. NaviBoard and NaviChair: Limited Translation Combined with Full Rotation for Efficient Virtual Locomotion. IEEE TVCG 2019, 27, 165-177. [CrossRef]

15. Zielasko, D.; Law, Y.C.; Weyers, B. Take a Look Around-The Impact of Decoupling Gaze and Travel-direction in Seated and Ground-based Virtual Reality Utilizing Torso-directed Steering. In Proceedings of the IEEE Conference on Virtual Reality and 3D User Interfaces (VR), Atlanta, GA, USA, 22-26 March 2020.

16. Dennison, M.S.; D'Zmura, M. Cybersickness Without the Wobble: Experimental Results Speak Against Postural Instability Theory. Appl. Ergon. 2017, 58, 215-223. [CrossRef]

17. Zielasko, D. Subject 001-A Detailed Self-Report of Virtual Reality Induced Sickness. In Proceedings of the IEEE VR Workshop on Immersive Sickness Prevention, Lisbon, Portugal, 28 March 2021.

18. Zielasko, D.; Horn, S.; Freitag, S.; Weyers, B.; Kuhlen, T.W. Evaluation of Hands-Free HMD-Based Navigation Techniques for Immersive Data Analysis. In Proceedings of the IEEE Symposium on 3D User Interfaces (3DUI), Greenville, SC, USA, 19-20 March 2016; pp. 113-119.

19. Hashemian, A.; Lotfaliei, M.; Adhikari, A.; Kruijff, E.; Riecke, B. HeadJoystick: Improving Flying in VR using a Novel Leaning-Based Interface. IEEE TVCG 2020. [CrossRef] [PubMed]

20. Bos, J.E.; Bles, W.; Groen, E.L. A Theory on Visually Induced Motion Sickness. Displays 2008, 29, 47-57. [CrossRef]

21. Kemeny, A.; George, P.; Mérienne, F.; Colombet, F. New VR Navigation Techniques to Reduce Cybersickness. Electron. Imaging 2017, 2017, 48-53.

22. Farmani, Y.; Teather, R.J. Evaluating Discrete Viewpoint Control to Reduce Cybersickness in Virtual Reality. Virtual Real. 2020, 24, 1-20. [CrossRef] 
23. Sra, M.; Jain, A.; Maes, P. Adding Proprioceptive Feedback to Virtual Reality Experiences Using Galvanic Vestibular Stimulation. In Proceedings of the ACM CHI Conference on Human Factors in Computing Systems, Glasgow, UK, 4-9 May 2019; pp. 1-14.

24. Chester, M.R.; Rys, M.J.; Konz, S.A. Leg Swelling, Comfort and Fatigue When Sitting, Standing, and Sit/Standing. Int. J. Ind. Ergon. 2002, 29, 289-296. [CrossRef]

25. Garcia, M.G.; Wall, R.; Steinhilber, B.; Läubli, T.; Martin, B.J. Long-Lasting Changes in Muscle Twitch Force During Simulated Work While Standing or Walking. Hum. Factors 2016, 58, 1117-1127. [CrossRef]

26. Zielasko, D.; Riecke, B.E. Either Give Me a Reason to Stand or an Opportunity to Sit in VR. In Proceedings of the IEEE VR Abstracts and Workshops, Atlanta, GA, USA, 22-26 March 2020; pp. 283-284. [CrossRef]

27. Balasubramanian, V.; Adalarasu, K.; Regulapati, R. Comparing Dynamic and Stationary Standing Postures in an Assembly Task. Int. J. Ind. Ergon. 2009, 39, 649-654. [CrossRef]

28. Gao, B.; Mai, Z.; Tu, H.; Duh, H.B.L. Evaluation of Body-centric Locomotion with Different Transfer Functions in Virtual Reality. In Proceedings of the IEEE Virtual Reality and 3D User Interfaces (VR), Lisbon, Portugal, 28 March 2021.

29. LaViola, J.J., Jr.; Kruijff, E.; McMahan, R.P.; Bowman, D.; Poupyrev, I.P. 3D User Interfaces: Theory and Practice; Addison-Wesley Professional: New York, NY, USA, 2017.

30. Cieślik, B.; Serweta, A.; Federico, S.; Szczepańska-Gieracha, J. Altered postural stability in elderly women following a single session of head-mounted display virtual reality. Acta Bioeng. Biomech. 2021, 23, 107-111. [CrossRef]

31. Wu, F.; Rosenberg, E.S. Combining Dynamic Field of View Modification with Physical Obstacle Avoidance. In Proceedings of the IEEE VR Workshop on Immersive Sickness Prevention, Osaka, Japan, 23-27 March 2019.

32. Simeone, A.L.; Velloso, E.; Gellersen, H. Substitutional Reality: Using the Physical Environment to Design Virtual Reality Experiences. In Proceedings of the 33rd Annual ACM Conference on Human Factors in Computing Systems, Seoul, Korea, 18-23 April 2015; pp. 3307-3316.

33. Zielasko, D.; Weyers, B.; Kuhlen, T.W. A Non-Stationary Office Desk Substitution for Desk-Based and HMD-Projected Virtual Reality. In Proceedings of the IEEE VR Workshop on Immersive Sickness Prevention, Osaka, Japan, $23-27$ March 2019.

34. Sra, M.; Garrido-Jurado, S.; Schmandt, C.; Maes, P. Procedurally Generated Virtual Reality from 3D Reconstructed Physical Space. In Proceedings of the 22nd ACM Conference on Virtual Reality Software and Technology, Munich, Germany, 2-4 November 2016; pp. 191-200. [CrossRef]

35. Hettinger, L.J.; Schmidt, T.; Jones, D.L.; Keshavarz, B. Illusory self-motion in virtual environments. In Handbook of Virtual Environments; Hale, K.S., Stanney, K.M., Eds.; CRC Press: Boca Raton, FL, USA, 2014; Chapter 18, pp. $435-466$.

36. Lawson, B.; Riecke, B.E. The perception of body motion. In Handbook of Virtual Environments: Design, Implementation, and Applications, 2nd ed.; Hale, K.S., Stanney, K.M., Eds.; CRC Press: Boca Raton, FL, USA, 2014; Chapter 7, pp. 163-195.

37. Riecke, B.E. Compelling self-motion through virtual environments without actual self-motion: Using self-motion illusions ("vection") to improve user experience in VR. In Virtual Reality; Kim, J.J., Ed.; InTechOpen: London, UK, 2011; Chapter 8, pp. 149-176. [CrossRef]

38. Riecke, B.E.; Schulte-Pelkum, J. An integrative approach to presence and self-motion perception research. In Immersed in Media: Telepresence Theory, Measurement and Technology; Biocca, F., Freeman, J., IJsselsteijn, W., Lombard, M., Schaevitz, R.J., Eds.; Springer: Berlin, Germany, 2015. [CrossRef]

39. Wong, S.C.P.; Frost, B.J. The effect of visual-vestibular conflict on the latency of steady-state visually induced subjective rotation. Percept. Psychophys. 1981, 30, 228-236. [CrossRef]

40. Schulte-Pelkum, J. Perception of Self-Motion: Vection Experiments in Multi-Sensory Virtual Environments. Ph.D. Thesis, Ruhr-Universität Bochum, Bochum, Germany, 2007.

41. Riecke, B.E. Simple user-generated motion cueing can enhance self-motion perception (Vection) in virtual reality. In Proceedings of the ACM Symposium on Virtual Reality Software and Technology, Limassol, Cyprus, 1-3 November 2005; pp. 104-107. [CrossRef]

42. Riecke, B.E.; Schulte-Pelkum, J.; Caniard, F.; Bülthoff, H.H. Towards Lean and Elegant Self-Motion Simulation in Virtual Reality. In Proceedings of the IEEE Conference on Virtual Reality, Bonn, Germany, 12-16 March 2005; pp. 131-138. [CrossRef]

43. Riecke, B.E.; Feuereissen, D. To move or not to move: Can active control and user-driven motion cueing enhance self-motion perception ("vection") in Virtual Reality? In Proceedings of the ACM Symposium on Applied Perception SAP, Los Angeles, CA, USA, 3-4 August 2012; pp. 17-24. [CrossRef]

44. Kruijff, E.; Marquardt, A.; Trepkowski, C.; Lindeman, R.W.; Hinkenjann, A.; Maiero, J.; Riecke, B.E. On Your Feet! Enhancing Vection in Leaning-Based Interfaces through Multisensory Stimuli. In Proceedings of the ACM Symposium on Spatial User Interaction, Tokyo, Japan, 15-16 October 2016, pp. 149-158. [CrossRef]

45. Riecke, B.E.; Trepkowski, C.; Kruijff, E. Human Joystick: Enhancing Self-Motion Perception (Linear Vection) by using Upper Body Leaning for Gaming and Virtual Reality. iSpaceLab Tech. Rep. 2016, 2016, 1-12.

46. Riecke, B.E.; Feuereissen, D.; Rieser, J.J. Auditory self-motion simulation is facilitated by haptic and vibrational cues suggesting the possibility of actual motion. ACM Trans. Appl. Percept. TAP 2009, 6, 20:1-20:22. [CrossRef]

47. Riecke, B.E. Cognitive and higher-level contributions to illusory self-motion perception (vection): Does the possibility of actual motion affect vection? Jpn. J. Psychon. Sci. 2009, 28, 135-139.

48. Lepecq, J.C.; Giannopulu, I.; Baudonniere, P.M. Cognitive effects on visually induced body motion in children. Perception 1995, 24, 435-449. [CrossRef] [PubMed] 
49. Kitson, A.; Riecke, B.E.; Hashemian, A.M.; Neustaedter, C. NaviChair: Evaluating an Embodied Interface Using a Pointing Task to Navigate Virtual Reality. In Proceedings of the ACM Symposium on Spatial User Interaction, Los Angeles, CA, USA, 8-9 August 2015; p. 123-126. [CrossRef]

50. Marchal, M.; Pettré, J.; Lécuyer, A. Joyman: A Human-Scale Joystick for Navigating in Virtual Worlds. In Proceedings of the IEEE Symposium on 3D User Interfaces (3DUI), Singapore, 19-20 March 2011; pp. 19-26. [CrossRef]

51. Riecke, B.E.; Zielasko, D. Towards an Affordance of Embodied Locomotion Interfaces in VR: How to Know How to Move? In Proceedings of the IEEE VR Abstracts and Workshops, Atlanta, GA, USA, 22-26 March 2020; pp. 295-296. [CrossRef]

52. Zielasko, D.; Riecke, B.E. Can We Give Seated Users in Virtual Reality the Sensation of Standing or Even Walking? Do We Want To? In Proceedings of the IEEE VR Abstracts and Workshops, Atlanta, GA, USA, 22-26 March 2020; pp. 281-282. [CrossRef]

53. Drury, C.; Hsiao, Y.; Joseph, C.; Joshi, S.; Lapp, J.; Pennathur, P. Posture and Performance: Sitting vs. Standing for Security Screening. Ergonomics 2008, 51, 290-307. [CrossRef] [PubMed]

54. Coomer, N.; Ladd, J.; Williams, B. Virtual Exploration: Seated versus Standing. In Proceedings of the Joint Conference on Computer Vision, Imaging and Computer Graphics Theory and Applications, Funchal, Madeira, Portugal, 27-29 January 2018; pp. 264-272. [CrossRef]

55. Brachtendorf, K.; Weyers, B.; Zielasko, D. Towards Accessibility in VR-Development of an Affordable Motion Platform for Wheelchairs. In Proceedings of the IEEE VR Abstracts and Workshops, Atlanta, GA, USA, 22-26 March 2020; pp. $291-292$. [CrossRef]

56. Ferdous, S.M.S. Improve Accessibility of Virtual and Augmented Reality for People With Balance Impairments. In Proceedings of the IEEE Virtual Reality (VR), Los Angeles, CA, USA, 18-22 March 2017; pp. 421-422. [CrossRef]

57. Parrish, K. Stand Up or Sit Down? Many Don't Take Advantage of VR's Room-Scale Experience. 2018. Available online: https:/ / www.digitaltrends.com/computing/oculus-rift-owners-want-to-sit-for-vr-experiences/ (accessed on 20 April 2021). 